\title{
Endophthalmitis in the Tertiary Referral Center in Iran; Etiology and Causative Organisms
}

\author{
Hamidreza Torabi ${ }^{*}$, Mohammad Riazi Esfahani², Seyed-Ali Tabatabai ${ }^{2}$ \\ 'Department of Ophthalmology, Baqiyatallah University of Medical Sciences, Tehran, Iran \\ ${ }^{2}$ Eye Research Center, Farabi Eye Hospital, Tehran University of Medical Sciences, Tehran, Iran
}

*Corresponding Author: Hamidreza Torabi, M.D., Assistant Professor of Ophthalmology, Department of Ophthalmology, Baqiyatallah University of Medical Sciences, Mollasadra Street, Vanak square, Tehran, Iran. Tel: +98-9124274780, Email: Dr_Hamidrezatorabi@yahoo.com

Received May 7, 2017; Accepted July 27, 2017; Online Published August 8, 2017

\begin{abstract}
Background: Endophthalmitis is a severe and sight-threatening complication of ocular surgery or ocular trauma. The identification of common types and causative organisms is essential for prevention and management.

Objective: The current study describes the prevalence of various types of endophthalmitis and the causative microbiological spectrum of each type treated in the Tertiary Referral Center in Iran.

Methods: All cases of endophthalmitis admitted to the Emergency Department of Farabi Eye hospital (Tehran, Iran) between July 2013 and December 2014 were included in this study. The patients' demographic data, type of endophthalmitis, aqueous or vitreous culture results, and treatment methods were recorded.

Results: From 108 presumed endophthalmitis cases, post-operative endophthalmitis $(68.5 \%)$ was the most frequent type followed by post-traumatic (25\%), bleb-associated (4.6\%), and endogenous (1.9\%) types. Streptococcus viridians (37.5\%) was the most common isolated organism in post-operative cases. In post-traumatic endophthalmitis patients, the most frequent causative organism was Staphylococcus epidermidis (70\%).

Conclusion: Many studies from other countries have reported that $S$. epidermidis is the leading cause of endophthalmitis after either intraocular surgeries or open-globe injuries, but the current study has shown that $S$. viridians is the most common isolated organism in post-operative endophthalmitis.

Keywords: Endophthalmitis, Post-cataract Endophthalmitis, Posttraumatic Endophthalmitis, Bleb-Associated Endophthalmitis, Vitreous Culture
\end{abstract}

\section{Background}

Endophthalmitis is an uncommon, but catastrophic complication of intraocular surgeries and open-globe injuries. Various types of intraocular surgeries may cause endophthalmitis, but improvements in aseptic and microsurgical techniques and the use of antibiotics has reduced the incidence of endophthalmitis. ${ }^{1,2}$

The incidence of acute-onset endophthalmitis after cataract surgery has been estimated to be $0.07 \%$ to $0.26 \%,{ }^{3}$ and it occurs in approximately $4 \%$ to $8 \%$ of eyes with open-globe injury. ${ }^{4-6}$ Some studies have reported a much wider variation in incidence rates of endophthalmitis following traumatic injury (4\% to $17 \%) .^{7-11}$ In Iran, the frequency of endophthalmitis was reported as $0.023 \%$ after cataract surgery ${ }^{12}$ and $2.1 \%$ after open-globe injury. ${ }^{13}$ Likewise, a wide variation in incidence of bleb-associated endophthalmitis, from $0.17 \%$ to $13.2 \%$, has been reported. ${ }^{14-29}$

Considering these wide fluctuations in incidence rates of various types of endophthalmitis and the obvious difference among them, this study aimed to determine the prevalence of various types of endophthalmitis and the causative organism in Iran's Tertiary Referral Center.

\section{Objective}

The current study aimed to determine the frequency of different types of endophthalmitis and the most common causative organisms in the Tertiary Referral Center of Iran.

\section{Methods}

In this prospective observational case series, approved by the local Ethics Review Committee of Tehran University of Medical Sciences, all patients admitted to Farabi Eye Hospital (Tehran, Iran) between July 2013 and December 2014 were included; no case was excluded. An initial diagnosis of endophthalmitis was ascertained clinically based on the presence of decreased vision, loss of red reflex, marked fibrinoid anterior chamber reaction or hypopyon,

Copyright $\odot 2017$ The Author(s). This is an open-access article distributed under the terms of the Creative Commons Attribution License (http:// creativecommons.org/licenses/by/4.0), which permits unrestricted use, distribution, and reproduction in any medium, provided the original work is properly cited. 
vitreous cells, or marked vitreous consolidation on B-scan ultrasonography.

Each patient's age and gender, type of endophthalmitis, aqueous or vitreous culture results, and treatment method were recorded.

Post-cataract surgery endophthalmitis cases were treated based on the Endophthalmitis Vitrectomy Study results. ${ }^{30}$ Patients with an initial visual acuity of light perception underwent a pars plana vitrectomy, vitreous sampling, and intravitreal injection of $1 \mathrm{mg}$ per $0.1 \mathrm{~mL}$ vancomycin and $2.25 \mathrm{mg}$ per $0.1 \mathrm{mg}$ ceftazidim. Aqueous and vitreous aspiration and intravitreal antibiotic injection (1 mg per $0.1 \mathrm{ml}$ vancomycin and $2.25 \mathrm{mg}$ per $0.1 \mathrm{mg}$ ceftazidim) were performed for patients with a visual acuity of hand motion or higher.

Aqueous or vitreous samples obtained by either needle tapping or pars plana vitrectomy were processed for direct smear using Gram and Giemsa stains. Specimens were inoculated onto blood agar, chocolate agar, and thioglycolate broth and incubated at $37^{\circ} \mathrm{C}$. Furthermore, cultures on Sabouraud agar at room temperature were used for fungal isolates. Descriptive statistics were used to present quantitative descriptions in this study.

\section{Results}

A total of 108 patients had clinical presentation of endophthalmitis and were admitted to Farabi Eye hospital during the study period. The mean patient age was $54.7 \pm 19$ years. Endophthalmitis involved the left eye in $61.1 \%$ of cases, and the right eye was involved in $38.9 \%$ of cases.

The most common type of endophthalmitis was postoperative $(68.5 \%)$; other less frequent types included posttraumatic (25\%), bleb-associated (4.6\%), and endogenous (1.9\%). The demographic data of study subjects is shown in Table 1.

Positive culture results were found in $43.5 \%$ of patients. Hypopyon was absent in $23.1 \%$ of patients. The culture positivity rate in patients with hypopyon was $49.4 \%$, but only $24 \%$ of patients without hypopyon had positive culture results.

\subsection{Postoperative Endophthalmitis}

The mean age of patients with postoperative endophthalmitis was $63.4 \pm 14$ years, and $58.1 \%$ of cases were men. All patients had unilateral endophthalmitis, and the left eye was affected in $66.2 \%$ of cases.

Postoperative endophthalmitis in 68 patients (91.9\%) was acute (less than 6 weeks from surgery to presentation) and was chronic in 6 patients (8.1\%). All chronic cases had occurred after cataract surgery. The mean time between surgery and presentation to the Tertiary Referral Center was $8.1 \pm 7$ days for acute cases.

The most frequent causative factor for surgery was cataract occurring in 65 patients $(87.8 \%)$. Other etiologic surgeries included a pars plana vitrectomy in 4 patients (5.4\%), intravitreal bevacizumab injection in 2 patients (2.7\%), phakic IOL implantation in 2 patients $(2.7 \%)$, and silicone oil removal in one patient (1.4\%).

Culture results were positive in 32 cases (43.2\%), and the most commonly isolated organism was Gram positive bacteria in 23 cases (71.9\% of culture positive cases). Among the bacteria, Streptococcus viridans was the most common isolate (Table 2).

\subsection{Posttraumatic Endophthalmitis}

The mean age of 27 patients with post-traumatic endophthalmitis was $31.4 \pm 14$ years. Twenty-five patients $(88.9 \%)$ were men. The right eye was affected in $51.9 \%$ of cases. The mean time between trauma and presentation was $3.6 \pm 3$ days.

Culture results were positive in 10 eyes (37\%), and Staphylococcus epidermidis was the most common isolate (Table 3). Intraocular foreign bodies were present in 15 eyes $(55.6 \%)$.

\subsection{Other Types of Endophthalmitis}

Culture results were positive in 3 of 5 cases with blebassociated endophthalmitis. S. viridians was isolated in two cases, and Candida albicans was isolated in one other patient.

Both patients with endogenous endophthalmitis had undetermined collagen vascular disease and were treated using systematic corticosteroids. Culture results were positive in both of them. Haemophilus sp. was isolated in one case and $C$. albicans was isolated in the other.

\section{Discussion}

The most common type of endophthalmitis in the current study was post-operative endophthalmitis with cataract surgery most frequently contributing to this condition. Somani et $\mathrm{al}^{31}$ (Canada), Pei-Chang et $\mathrm{al}^{32}$ (Taiwan), and Falavarjani et $\mathrm{al}^{33}$ (Iran) reported that cataract surgery was the most common predisposing surgical procedure in postoperative endophthalmitis patients. Since cataract surgery is the most common intraocular surgery, most cases of post-operative endophthalmitis occur after cataract extraction. ${ }^{34}$

In the current study it was noted that $66.2 \%$ of post-

Table 1. Demographic Data of 108 Study Subjects

\begin{tabular}{lcccc}
\hline & $\begin{array}{c}\text { Postcataract } \\
\text { Endophthalmitis, } \mathbf{n = 7 4}\end{array}$ & $\begin{array}{c}\text { Posttraumatic } \\
\text { Endophthalmitis, } \mathbf{n}=\mathbf{2 7}\end{array}$ & $\begin{array}{c}\text { Bleb-Associated } \\
\text { Endophthalmitis, } \mathbf{n = 5}\end{array}$ \\
\hline Males (\%) & 58.1 & 88.9 & 20 & 0 \\
Females (\%) & 41.9 & 11.1 & 80 & 100 \\
Mean age \pm SD (y) & $63.4 \pm 14$ & $31.4 \pm 14$ & $66 \pm 11$ & 39 \\
Incidence (\%) & 68.5 & 25 & 4.6 & 1.9 \\
\hline
\end{tabular}

Abbreviation: SD, standard deviation. 
Table 2. Microbiological Spectrum in Patients With Postoperative Endophthalmitis

\begin{tabular}{lcc}
\hline Organism & Number & Percent \\
\hline Gram positive & 12 & 37.5 \\
Streptococcus viridans & 7 & 21.9 \\
Staphylococcus epidermidis & 2 & 6.3 \\
Staphylococcus aureus & 1 & 3.1 \\
Streptococcus pneumoniae & 1 & 3.1 \\
Entrococcus sp. & & \\
Gram negative & 4 & 12.5 \\
Pseudomonas aeruginosa & 1 & 3.1 \\
Haemophilus sp. & 1 & 3.1 \\
Escherichia coli & 1 & 3.1 \\
Stenotrophomonas moltiphilia & 1 & 3.1 \\
Enterobacteriaceae sp. & & \\
Fungi & 1 & 3.1 \\
Fusarium sp. &
\end{tabular}

Table 3. Microbiological Spectrum in Patients With Posttraumatic Endophthalmitis

\begin{tabular}{lcc}
\hline Organism & Number & Percent \\
\hline Gram positive & 7 & 70 \\
Staphylococcus epidermidis & 1 & 10 \\
Bacillus cereus & & \\
Gram negative & 1 & 10 \\
Pseudomonas aeruginosa & 1 & 10 \\
Enterobacteriaceae sp. & \\
\hline
\end{tabular}

operative endophthalmitis cases occurred in the left eye. This result is not in agreement with Hani et $\mathrm{al}^{3}$ and Miller et $\mathrm{al}^{35}$ who reported that most cases occur in the right eye. They believed that the characteristics of corneal incisions performed by right-handed surgeons may contribute to the higher prevalence of endophthalmitis in the right eye. However, the hand dominance of surgeons was not described in this report; thus, the higher prevalence of endophthalmitis in the right eye may not be correct.

The culture positivity rate in patients with postoperative endophthalmitis was $43.2 \%$ in this study, which is lower than previously reported, such as the Endophthalmitis Vitrectomy Study that had 67\% culture-positive cases ${ }^{9}$ and other series seen in the Netherlands (66.4\%), ${ }^{36}$ India (52.6\%), ${ }^{37}$ and Taiwan (55\%). ${ }^{32}$ Furthermore, a retrospective study in China recorded a culture-positive rate of $44.2 \%{ }^{38}$

Additionally, the current study detected positive cultures in $37 \%$ of patients with posttraumatic endophthalmitis, which is lower than other reports such as in Saudi Arabia (63.2\%). ${ }^{39}$ This discrepancy may be due in some part to the method of sample collection which was performed by needle aspiration in most cases in the current study. In patients with endophthalmitis, the vitreous body contains inflammatory condensations; so, specimens obtained through needle aspiration may not be sufficient for analysis. Also, the lower culture positivity rate in the current study may be attributable to inappropriate sample collection or handling or the higher frequency of sterile endophthalmitis in the series of this study.

In a retrospective study, Melo et al reported that only
$42 \%$ of all presumed endophthalmitis cases have had culture-positive results, and most of culture-positive cases were secondary to surgical procedures $(62 \%) .^{40}$

In the current study, S. viridans was found to be the most frequent cause of postoperative endophthalmitis (37.5\%) followed by S. epidermidis (21.9\%) and Pseudomonas aeruginosa (12.5\%) as the second and third most common organisms, respectively.

In their report, Hani et al. (Saudi Arabia) isolated $S$. viridans in one case (from 18 cases) of postoperative endophthalmitis, and S. epidermidis was the most frequent causative organism. Pei-Chang et a ${ }^{32}$ also reported that $S$. epidermidis was the most frequent organism causing postoperative endophthalmitis. Furthermore, Zhu et al reported that $S$. epidermidis was the most common isolated organism in culture-positive post-operative endophthalmitis cases. ${ }^{38}$ Therefore, some geographic variations may contribute to the prevalence of $S$. viridians infection in Iran.

The most common organism identified in patients with post-traumatic endophthalmitis in the current study was $S$. epidermidis (70\%). This result is compatible with those of Abdulrahman et al ${ }^{39}$ and Abu el-Asrar et al. ${ }^{41}$

Hani et $\mathrm{al}^{3}$ reported a lack of hypopyon in $30 \%$ of cases. Hypopyon was lacking in $23.1 \%$ of cases in the current study. The culture positivity rate was higher in patients who had hypopyon in the initial examination. Thus, the presence of hypopyon at presentation is a strong predictor of positive culture results.

Intraocular foreign bodies (IOFB) were present in 55.6\% of posttraumatic endophthalmitis cases in the current study. IOFB were reported in $43 \%$ of cases in one study ${ }^{39}$ and in $50 \%$ of cases in another. ${ }^{41}$ Education for high risk occupational groups and use of protective eye wears are important factors in the prevention of open-globe injuries and trauma-associated endophthalmitis.

\section{Conclusion}

There appears to be a wide variation in endophthalmitis incidence rates; therefore, this study was conducted. Postcataract surgery endophthalmitis is the most frequently seen type of endophthalmitis in the Tertiary Referral Center of Iran. In contrast to many previous reports from other countries, $S$. viridans is the most common organism contributing to culture-proven endophthalmitis in post-operative patients at this center. Furthermore, $S$. epidermidis is the most common microbiologic cause of post-traumatic endophthalmitis in this study.

\section{Authors' Contributions}

Study Design: HT and MRE; data collection: HT; data analysis: HT; manuscript writing: HT; Supervision: MRE and SAT.

\section{Conflict of Interest Disclosures}

No conflicts of interest are declared.

\section{Ethical Approval}

The current study was approved by the local Ethics Review Committee of Tehran University of Medical Sciences. 


\section{Research Highlights}

\section{What Is Already Known?}

Cataract surgery is the most common type of operation that may cause post-operative endophthalmitis. The most frequently isolated organisms in both post-operative and post-traumatic endophthalmitis cases were S. epidermidis in most previous studies.

\section{What This Study Adds?}

Like studies from many other countries, the most frequent causative surgery in post-operative endophthalmitis cases was cataract surgery in the Tertiary Referral Center; S. viridans, however, was the most common isolated organism in such cases. Also, like other reports, S. epidermidis was the most frequent organism to cause post-traumatic endophthalmitis in this center.

\section{References}

1. Eifrig CW, Flynn HW Jr, Scott IU, Newton J. Acute-onset postoperative endophthalmitis: review of incidence and visual outcomes (1995-2001). Ophthalmic Surg Lasers. 2002;33:373-378.

2. Li J, Morlet N, Ng JQ, Semmens JB, Knuiman MW. Significant nonsurgical risk factors for endophthalmitis after cataract surgery: EPSWA Fourth Report. Invest Ophthalmol Vis Sci. 2004;45:1321-1328. doi:10.1167/iovs.03-1000.

3. Al-Mezaine HS, Kangave D, Al-Assiri A, Al-Rajhi AA. Acuteonset nosocomial endophthalmitis after cataract surgery Incidence, clinical features, causative organisms, and visual outcomes.J Cataract Refract Surg. 2009;35(4):643-649. doi:10.1016/j.jcrs.2009.01.003.

4. Thompson WS, Rubsamen PE, Flynn HW Jr, Schiffman J, Cousins SWI. Endophthalmitis after penetrating trauma. Risk factors and visual acuity outcomes. Ophthalmology. 1995;102:1696-701. doi:10.1016/S0161-6420(95)30807-X.

5. Duch-SamperAM,MenezoJL,Hurtado-SarrioM. Endophthalmitis following penetrating eye injuries. Acta Ophthalmol Scand. 1997;75:104-106. doi:10.1111/j.1600-0420.1997.tb00263.x.

6. Thompson JT, Parver LM, Enger CL, Mieler WF, Liggett PE. Infectious endophthalmitis after penetrating injuries with retained intraocular foreign bodies. National Eye Trauma System. Ophthalmology. 1993;100:1468-1474. doi:10.1016/ S0161-6420(93)31454-5.

7. Brinton GS, Topping TM, Hyndiuk RA, Aaberg TM, Reeser FH, Abrams GW. Posttraumatic endophthalmitis. Arch Ophthalmol. 1984;102(4):547-550. doi:10.1001/ archopht.1984.01040030425016.

8. Boldt HC, Pulido JS, Blodi CF, Folk JC, Weingeist TA. Rural endophthalmitis. Ophthalmology. 1989;96(12):1722-1726. doi:10.1016/S0161-6420(89)32658-3.

9. Verbraeken $\mathrm{H}$, Rysselaere M. Post-traumatic endophthalmitis. Eur J Ophthalmol. 1994;4(1):1-5

10. Sabaci G, Bayer A, Mutlu FM, Karagül S, Yildirim E. Endophthalmitis after deadly-weapon-related open-globe injuries: risk factors, value of prophylactic antibiotics, and visual outcomes. Am J Ophthalmol. 2002;133(1):62-69. doi:10.1016/S0002-9394(01)01320-4

11. Essex RW, Yi Q, Charles PG, Allen PJ. Post-traumatic endophthalmitis. Ophthalmology. 2004;111(11):2015-2022. doi:10.1016/j.ophtha.2003.09.041.

12. Jabbarvand $M$, Hashemian $H$, Khodaparast $M$, Jouhari $M$, Tabatabaei A, Rezaei S. Endophthalmitis occurring after cataract surgery: outcomes of more than 480000 cataract surgeries, epidemiologic features, and risk factors. Ophthalmology. 2016;123(2):295-301. doi:10.1016/j.ophtha.2015.08.023.
13. Dehghani AR, Rezaei L, Salam H, Mohammadi Z, Mahboubi M. Post traumatic endophthalmitis: incidence and risk factors. Glob J Health Sci. 2014;6(6):68-72. doi:10.5539/gihs.v6n6p68.

14. Sharan S, Trope GE, Chipman M, Buys YM. Late-onset bleb infections: prevalence and risk factors. Can J Ophthalmol. 2009;44(3):279-83. doi:10.3129/i09-050.

15. Alwitry A, King AJ. Surveillance of late-onset bleb leak, blebitis and bleb-related endophthalmitis--a UK incidence study. Graefes Arch Clin Exp Ophthalmol. 2012;250(8):1231-1236. doi:10.1007/s00417-011-1920-5.

16. DeBry PW, Perkins TW, Heatley G, Kaufman P, Brumback LC. Incidence of late-onset bleb-related complications following trabeculectomy with mitomycin. Arch Ophthalmol. 2002;120(3):297-300. doi:10.1001/archopht.120.3.297.

17. Greenfield DS, Suñer IJ, Miller MP, Kangas TA, Palmberg PF, Flynn HW Jr. Endophthalmitis after filtering surgery with mitomycin. Arch Ophthalmol. 1996;114(8):943-9. doi:10.1001/archopht.1996.01100140151007.

18. Katz LJ, Cantor LB, Spaeth GL. Complications of surgery in glaucoma. Early and late bacterial endophthalmitis following glaucoma filtering surgery. Ophthalmology. 1985;92(7):959963. doi:10.1016/S0161-6420(85)33948-9.

19. Freedman J, Gupta M, Bunke A. Endophthalmitis after trabeculectomy. Arch Ophthalmol. 1978;96(6):1017-1018. doi:10.1001/archopht.1978.03910050541009.

20. Higginbotham EJ, Stevens RK, Musch DC, et al. Bleb-related endophthalmitis after trabeculectomy with mitomycin C. Ophthalmology. 1996;103(4):650-6. doi:10.1016/S0161_ 6420(96)30639-8.

21. Wolner B, Liebmann JM, Sassani JW, Ritch R, Speaker M, Marmor M. Late bleb-related endophthalmitis after trabeculectomy with adjunctive 5-fluorouracil. Ophthalmology. 1991;98(7):10531060. doi:10.1016/S0161-6420(91)32177-8.

22. Ticho $U$, Ophir A. Late complications after glaucoma filtering surgery with adjunctive 5-fluorouracil. Am J Ophthalmol 1993:115(4):506-10. doi:10.1016/S0002-9394(14)74454-X.

23. Solomon A, Ticho U, Frucht-Pery J. Late-onset, bleb-associated endophthalmitis following glaucoma filtering surgery with or without antifibrotic agents. J Ocul Pharmacol Ther. 1999;15(4):283-293. doi:10.1089/jop.1999.15.283.

24. Mochizuki K, Jikihara S, Ando Y, Hori N, Yamamoto T, Kitazawa Y. Incidence of delayed onset infection after trabeculectomy with adjunctive mitomycin $\mathrm{C}$ or 5 -fluorouracil treatment. $\mathrm{Br}$ J Ophthalmol. 1997;81(10):877-83. doi:10.1136/ bjo.81.10.877.

25. Mégevand GS, Salmon JF, Scholtz RP, Murray AD. The effect of reducing the exposure time of mitomycin $\mathrm{C}$ in glaucoma filtering surgery. Ophthalmology. 1995;102(1):84-90. doi:10.1016/S0161-6420(95)31049-4

26. Muckley ED, Lehrer RA. Late-onset blebitis/endophthalmitis: incidence and outcomes with mitomycin C. Optom Vis Sci. 2004;81(7):499-504. doi:10.1097/00006324-20040700000010.

27. Kuang TM, Lin YC, Liu CJ, Hsu WM, Chou CK. Early and late endophthalmitis following trabeculectomy in a Chinese population. Eur J Ophthalmol. 2008;18(1):66-70.

28. Wallin Ö, Al-ahramy AM, Lundström M, Montan P. Endophthalmitis and severe blebitis following trabeculectomy. Epidemiology and risk factors; a single-centre retrospective study. Acta Ophthalmol. 2014;92(5):426-431. doi:10.1111/ aos.12257.

29. Yamamoto T, Sawada A, Mayama C, et al. The 5-year incidence of bleb-related infection and its risk factors after filtering surgeries with adjunctive mitomycin C: collaborative bleb-related infection incidence and treatment study 2. Ophthalmology. 2014;121(5):1001-6. doi:10.1016/j.ophtha.2013.11.025

30. Endophthalmitis Vitrectomy Study Group. Results of the Endophthalmitis Vitrectomy Study: a randomized trial of immediate vitrectomy and of intravenous antibiotics for the treatment of postoperative bacterialendophthalmitis. Arch Ophthalmol. 1995;113:1479-1496. doi:10.1001/ 
archopht.1995.01100120009001.

31. Somani S, Grinbaum A, Slomovic AR. Postoperative endophthalmitis: incidence, predisposing surgery, clinical course and outcome. Can J Ophthalmol. 1997;32(5):303-310.

32. Wu PC, Kuo HK, Li M, et al. Nosocomial postoperative endophthalmitis: a 14-year review. Graefes Arch Clin Exp Ophthalmol. 2006;244(8):920-9. doi:10.1007/s00417-0050170-9.

33. Falavarjani KG, Nekoozadeh $S$, Modarres $M$, et al. Isolates and antibiotic resistance of culture-proven endophthalmitis cases presented to a referral center in Tehran. Middle East Afr J Ophthalmol. 2012;19(4):361-363. doi:10.4103/09749233.102740.

34. Verbraeken HE. Endophthalmitis after lens extraction. Doc Ophthalmol. 1994;87:153-158. doi:10.1007/BF01204792.

35. Miller JJ, Scott IU, Flynn HW Jr, Smiddy WE, Newton J, Miller D. Acute-onset endophthalmitis after cataract surgery (2000-2004): incidence, clinical settings, and visual acuity outcomes after treatment. Am J Ophthalmol. 2005;139:983987. doi:10.1016/j.ajo.2005.01.025.

36. Pijl BJ, Theelen T, Tilanus MA, Rentenaar R, Crama N. Acute endophthalmitis after cataract surgery: 250 consecutive cases treated at a tertiary referral center in the Netherlands.
Am J Ophthalmol. 2010;149(3):482-7.e1-2. doi:10.1016/j. ajo.2009.09.021.

37. Lalitha P, Rajagopalan J, Prakash K, Ramasamy K, Prajna NV Srinivasan M. Postcataract endophthalmitis in South India incidence and outcome. Ophthalmology. 2005;112(11): 188590. doi:10.1016/j.ophtha.2005.05.020.

38. Zhu Y, Chen X, Chen P, Wu J, Hua H, Yao K. The occurrence rate of acute-onset postoperative endophthalmitis after cataract surgery in Chinese small- and medium-scale departments of ophthalmology. Sci Rep. 2017;7:40776. doi:10.1038/ srep40776.

39. Al-Omran AM, Abboud EB, Abu El-Asrar AM. Microbiologic spectrum and visual outcome of posttraumatic endophthalmitis. Retina. 2007;27(2):236-242. doi:10.1097/01 iae.0000225072.68265.ee.

40. Melo GB, Bispo PJ, Yu MC, Pignatari AC, Höfling-Lima AL. Microbial profile and antibiotic susceptibility of culture-positive bacterial endophthalmitis. Eye (Lond). 2011;25(3):382-388. doi:10.1038/eye.2010.236

41. Abu el-Asrar AM, al-Amro SA, al-Mosallam AA, al-Obeidan S. Post-traumatic endophthalmitis: causative organisms and visual outcome. Eur J Ophthalmol. 1999;9(1):21-31. 